\title{
Efecto del envasado en atmosfera modificada sobre la conservación de la carne de ternera liofilizada
}

\author{
Effect of modified atmosphere packaging on frezze-dried beef meat conservation
}

\author{
Daisy Alice Vera y Aragón Quintero ${ }^{1 *}$ y María Jesús Cantalejo Díez ${ }^{2}$
}

${ }^{1}$ Facultad de Ciencias Agrarias, Universidad Nacional de Asunción. Campus San Lorenzo. San Lorenzo. Paraguay.
${ }^{2}$ Departamento Tecnología de Alimentos, Universidad Pública de Navarra. Campus Arrosadía. Pamplona. España.
$\begin{aligned} & * \text { Autor para correspondencia (daisy.verayaragon@agr.una.py) } \\ & \text { Recibido: 10/09/2015; Aceptado: 21/04/2016. } \\ & \text { Doi:10.18004/investig.agrar.2016.junio.15-21 }\end{aligned}$

\section{RESUMEN}

La carne liofilizada debidamente envasada puede ser almacenada por tiempo ilimitado, conservando la mayoría de sus propiedades físico-químicas y sensoriales semejantes a la carne fresca. La investigación consistió en envasar muestras de carne de ternera liofilizada con porcentaje de humedad residual inferior al 3\%, actividad de agua por debajo de 0,2 y porcentaje de rehidratación entre 95 y 100\%. El proceso de liofilización utilizado en el experimento fue el de aplicación de congelación rápida, $25 \mathrm{~Pa}$ de presión, $10 \mathrm{~h}$ de primer secado primario a $0^{\circ} \mathrm{C}$ y $3 \mathrm{~h}$ de segundo secado primario a $10^{\circ} \mathrm{C}$ de temperatura. Como el producto final debía conservar las propiedades por mucho tiempo y mantenerse estable microbiológicamente, se requirió de un envasado adecuado con baja permeabilidad a la transferencia al vapor de agua y al $\mathrm{O}_{2}$. Para ello se realizaron varios ensayos de envasado en atmosfera modificada con distintas mezclas y concentraciones de gases $\left(\mathrm{N}_{2}\right.$ y $\left.\mathrm{CO}_{2}\right)$ que favorecieron la conservación del producto, principalmente, el color, ya que es la propiedad física que se vio más afectada tras el proceso de liofilización. El valor de a* (tonalidad roja) de las muestras liofilizadas se mantuvo mejor en un envasado con una atmósfera de $80 \%$ $\mathrm{N}_{2}-20 \% \mathrm{CO}_{2}$ a lo largo del tiempo de almacenamiento. Sensorialmente, las muestras envasadas en $65 \% \mathrm{~N}_{2}-35 \%$ $\mathrm{CO}_{2}$ fueron más blandas.

Palabras clave: liofilización, Carne de ternera, envasado en atmosfera modificada.

\section{INTRODUCCIÓN}

El envasado de los alimentos tiene como función fundamental la protección del producto de posibles contaminaciones o recontaminaciones. Es por ello que el envasado tiene cinco funciones principales: contención del producto, conservación y calidad, presentación y

\begin{abstract}
Freeze-dried meat, when properly packaged, may be stored for unlimited time, preserving most of its physico chemical and sensory properties, like those of fresh meat. The research consisted on packaging freeze-dried meat samples with residual humidity content below $3 \%$, a water activity value below 0,2 and a rehydration percentage between 95 and 100\%. The freeze-drying process used was that of fast freezing application, a pressure of $25 \mathrm{~Pa}$, first primary drying for $10 \mathrm{~h}$ at $0^{\circ} \mathrm{C}$ and a second primary drying for $3 \mathrm{~h}$ at $10^{\circ} \mathrm{C}$. Since a condition is that the final product should keep its properties for a long time as well as remain microbiologically stable, a suitable packaging with low permeability to water vapour transfer and to $\mathrm{O}_{2}$ was required. To meet this requirement, several assays were carried out with packaging in modified atmosphere using different mixtures and gas concentrations $\left(\mathrm{N}_{2}, \& \mathrm{CO}_{2}\right)$ thus favoring product's features preservation, mainly colour which was the most affected characteristic following the lyophilization process. The value of $\mathrm{a}^{*}$ (redness) of the lyophilized samples, was best preserved in a $80 \% \quad \mathrm{~N}_{2}-20 \% \quad \mathrm{CO}_{2}$ atmosphere packaging over the storage period. Regarding meat's sensory properties, samples packaged in $65 \% \mathrm{~N}_{2}-$ $35 \% \mathrm{CO}_{2}$ atmosphere were the most tender ones.
\end{abstract}

Key words: freeze-dried or lyophilization, beef meat, modified atmosphere packaging.

comodidad, protección y extensión de la vida útil del producto (Shafiur Rahman 2007).

En cuanto al envasado en atmosfera modificada (EAM) este es un método que implica la eliminación del aire del 
interior del envase y su sustitución por un gas o mezcla de gases; la mezcla de gases a emplear depende del tipo de producto. La atmosfera gaseosa cambia continuamente durante todo el periodo de almacenamiento, por la influencia de diferentes factores, como respiración del producto envasado, cambios bioquímicos y la lenta difusión de los gases a través del envase (Parry 1995).

Para la conservación de la carne fresca, la composición de la atmósfera debe ser rica en oxígeno, ya que favorece la conservación del color rojo de la carne. En caso contrario, adquieren tonalidades pardas y grisáceas poco atractivas para el consumidor (García 2006).

A diferencia de la carne fresca, los productos curados y cocidos mantienen sus colores característicos si se elimina el oxígeno del espacio de cabeza. En presencia de este gas, los pigmentos responsables del color se oxidan dando lugar a compuestos verdes, amarillos o incoloros. Además, la carne y sus derivados se envasan con materiales de baja permeabilidad al oxígeno y a la humedad para evitar las reacciones de oxidación y los problemas de deshidratación (García 2006).

Por ejemplo, para evitar la decoloración rosa del jamón curado, el contenido inicial de $\mathrm{O}_{2}$ debería ser inferior al 0,5\% (Moller et al. 2000). Nannerup et al. (2004) observaron que el aumento de $\mathrm{O}_{2}$ lleva a una degradación del color rosa del jamón cocido. En otra investigación se concluyó que la reducción del nivel de iluminación puede evitar la decoloración (siempre y cuando el nivel de oxígeno sea bajo), pero en niveles de oxígeno por encima de, aproximadamente, 0,55\%, el jamón cocido sufrió decoloración, independientemente del nivel de iluminación (Moller et al. 2003).

El objetivo de la investigación fue analizar los efectos del envasado en atmosfera modificada sobre el pH, color, textura y atributos sensoriales de las muestras liofilizadas, almacenadas durante veintiocho días (28 días) a temperatura ambiente para determinar la mejor mezcla de gases a ser utilizada en la conservación de carne de ternera liofilizada.

\section{MATERIALES Y MÉTODOS}

Se utilizaron filetes de ternera de 4,65 $\pm 0,86 \mathrm{~mm}$ de espesor, procedentes del Matadero de Pamplona. La parte utilizada fue la babilla (corte español correspondiente a la cuadrada en el corte paraguayo). Los filetes fueron cortados en rectángulos pequeños de $4 \mathrm{~cm}$ de largo por 3 $\mathrm{cm}$ de ancho y posteriormente fueron colocadas en forma de cuadrícula en las bandejas de liofilizar. Para el proceso de liofilización se utilizó un liofilizador Telstar Lyobeta 25 a escala piloto. Dicho liofilizador está conectado al Lyostarlab que es un programa SCADA (Supervisión, control y adquisición de datos) especialmente diseñado y desarrollado para los procesos de liofilización de Telstar Industrial, S.L. Las condiciones del proceso de liofilización llevada a cabo fueron las siguientes: congelación rápida $(3,50 \mathrm{~h}),-45^{\circ} \mathrm{C}$ de temperatura de congelación, $0^{\circ} \mathrm{C}$ de temperatura de primer secado primario durante $10 \mathrm{~h}$, luego $10^{\circ} \mathrm{C}$ de temperatura de segundo secado primario durante 3 h y $25 \mathrm{~Pa}$ de presión.

Una vez finalizado el proceso de liofilización, las muestras fueron envasadas en atmosfera modificada para lo cual fueron utilizados dos gases diferentes: nitrógeno y dióxido de carbono, aplicados en distintas concentraciones y diferentes combinaciones (Tabla 1). Para ello se procedió a colocar las muestras en barquetas ovales H.50 de PP - EVOH - PP blanco 10/10 y posteriormente cerradas con film transparente P12 7060BXSTNP de PET 12 (PP - EVO - PP 70), con las siguientes características técnicas (datos suministrados por el fabricante - Tecnopack) :

- Espesor total: 82 micras (PET 12 micras, PP EVO - PP 70 micras)

- Permeabilidad al $\mathrm{O}_{2}:<1,5 \mathrm{ml} / \mathrm{m}^{2} / 24 \mathrm{~h} / \mathrm{bar}$

- Permeabilidad al vapor de agua: < 4,5 $\mathrm{g} / \mathrm{m}^{2} / 24 \mathrm{~h} / \mathrm{bar}$

Para el envasado se utilizó una envasadora ILPRA Systems - CE 98 termosadatrici FP 2387 Basic - con ayuda de un mezclador de gases PBI Dansensor MAP Mix 9000 - 3/150V (Gas mixer). Para las mediciones de gases se utilizó un lector de gases Sistech - instruments Gaspace 2 .

Tabla 1. Condiciones y combinaciones utilizadas para el envasado en atmosfera modificadas de las muestras de carne de ternera liofilizada.

\begin{tabular}{lccc}
\hline \multirow{2}{*}{ Procesos } & \multirow{2}{*}{ Tratamientos } & \multicolumn{2}{c}{$\begin{array}{c}\text { Condiciones de envasado } \\
(\boldsymbol{\%})\end{array}$} \\
\cline { 3 - 4 } & & $\mathbf{N}_{\mathbf{2}}$ & $\mathbf{C O}_{\mathbf{2}}$ \\
\hline \multirow{3}{*}{ Solo } & EL 1 & 100 & $(--)$ \\
Liofilizado & EL 2 & 80 & 20 \\
& EL 3 & 70 & 30 \\
& EL 4 & 65 & 35 \\
& EL 5 & 60 & 40 \\
\hline
\end{tabular}

EL: envasado liofilizado

Para denominar a los tratamientos se utilizaron las siglas EL (envasado liofilizado) y en cuanto al número presentado en subíndice correspondió a las 
concentraciones de gases aplicados: en primer lugar $\mathrm{N}_{2} \mathrm{y}$ en segundo lugar $\mathrm{CO}_{2}$ (ejemplo EL2 8020 ).

En el estudio comparativo fue necesario elaborar combinaciones crecientes de $\mathrm{CO}_{2}$ donde se analizó el efecto de éste gas sobre las muestras de ternera liofilizadas de los tratamientos EL1 100, EL2 80 20, EL3 70 30, EL465 $_{35}$ y EL560 40. Así mismo fue necesario medir la evolución de concentración de los gases a lo largo de los 28 días de almacenamiento.

Los análisis realizados fueron: $\mathrm{pH}$ : Se midió con un $\mathrm{pH}$ metro de punzón (Crison $\mathrm{PH} 25$, con electrodo de $\mathrm{pH}$ de penetración 52-32, pH: 2-11) sobre seis muestras de carne liofilizada rehidratada de cada tratamiento.

Color: El color de las muestras fue medido por reflexión, utilizando un espectrofotómetro portátil (Minolta CM2500d) y el espacio de color CIELab. El iluminante empleado fue el D65 y ángulo de observador $10^{\circ}$. Se analizaron los datos de L* (luminosidad), a* (rojo) y b* (amarillo). Para el análisis de carne liofilizada rehidratada se utilizaron seis muestras de cada tratamiento.

Textura: Con un Texturómetro TAXT2i (Aname) se registró la fuerza máxima $(\mathrm{N})$ de rotura utilizando un penetrómetro de cilindro de $12 \mathrm{~mm}$ de diámetro, aplicando $50 \mathrm{~N}$ con una distancia de $12 \mathrm{~mm}$ a una velocidad de ensayo de $1,0 \mathrm{~mm} / \mathrm{s}$. Las mediciones se realizaron en las muestras liofilizadas rehidratadas cocinadas (diez muestras) de cada tratamiento. La cocción de las muestras se realizó a $80^{\circ} \mathrm{C}$ en un baño termostático JUBALO, durante 2 min.

Descripción de las características sensoriales: Se realizaron análisis sensoriales descriptivos a cada tratamiento, utilizando muestras de carne de ternera liofilizadas (rehidratadas y cocinadas). Las muestras fueron cocinadas a $80^{\circ} \mathrm{C}$ en un baño termostático JUBALO, durante 2 min. Los análisis fueron realizados por un panel de cata entrenado del laboratorio (10 catadores). Cada catador tuvo a su disposición las muestras de forma codificada. Para evitar el error de tiempo y el efecto de contraste, se aplicó la técnica de permutación descrito en la sección de manejo de muestras por Pedrero y Pangborn (1989). Para ello, fueron utilizadas fichas de cata con una escala verbal de siete puntos. Los atributos evaluados fueron el aspecto, utilizando el modelo de ficha propuesto por Hunt et al. (1991), el perfil de textura para analizar el atributo dureza, utilizando el modelo de ficha propuesto por Lyon y Lyon (1991).

Los análisis de las propiedades químicas, físicas $\mathrm{y}$ sensoriales se realizaron los días $0,7,14,21$ y 28 .

\section{RESULTADOS Y DISCUSIÓN}

En la Figura 1 se observa la evolución de las diferentes concentraciones crecientes de $\mathrm{CO}_{2}$ utilizadas en las distintas atmosferas modificadas ensayadas, teniendo como control el comportamiento individual de las muestras envasadas en $100 \% \mathrm{~N}_{2}$.

En el caso del envasado con $\mathrm{N}_{2}$ se observó que este gas fue disminuyendo gradualmente a lo largo del tiempo de almacenamiento. Esto pudo deberse a que el $\mathrm{N}_{2}$ no llegó al $100 \%$ sino tan sólo al $98 \%$, y el $2 \%$ restante debió corresponder al $\mathrm{O}_{2}$ del ambiente que no fue totalmente eliminado durante el proceso de envasado y que, una vez dentro del envase y en contacto con el producto a lo largo del almacenamiento, fue aumentando debido a las características del producto liofilizado y a que el $\mathrm{N}_{2}$ no fue eficaz en su función como gas de arrastre del $\mathrm{O}_{2}$. Esto concuerda con Parry (1995) quien dice que la atmosfera gaseosa cambia continuamente durante todo el periodo de almacenamiento debido a varios factores.

Con relación a las combinaciones crecientes de $\mathrm{CO}_{2}$ se observó que, a medida que aumentaba la concentración de $\mathrm{N}_{2}$, disminuía gradualmente la concentración de $\mathrm{CO}_{2}$.

Fue analizado y comparado el efecto de las diferentes concentraciones de $\mathrm{CO}_{2}$ utilizadas en el envasado de las muestras liofilizadas.

En el caso del pH se observó que inicialmente el valor del $\mathrm{pH}$ de las muestras del tratamiento EL1 $1_{100}$ fue mucho más bajo que el $\mathrm{pH}$ de las muestras de los tratamientos EL2 80 20, EL370 30, EL465 35 y EL560 40 (Tabla 2). 


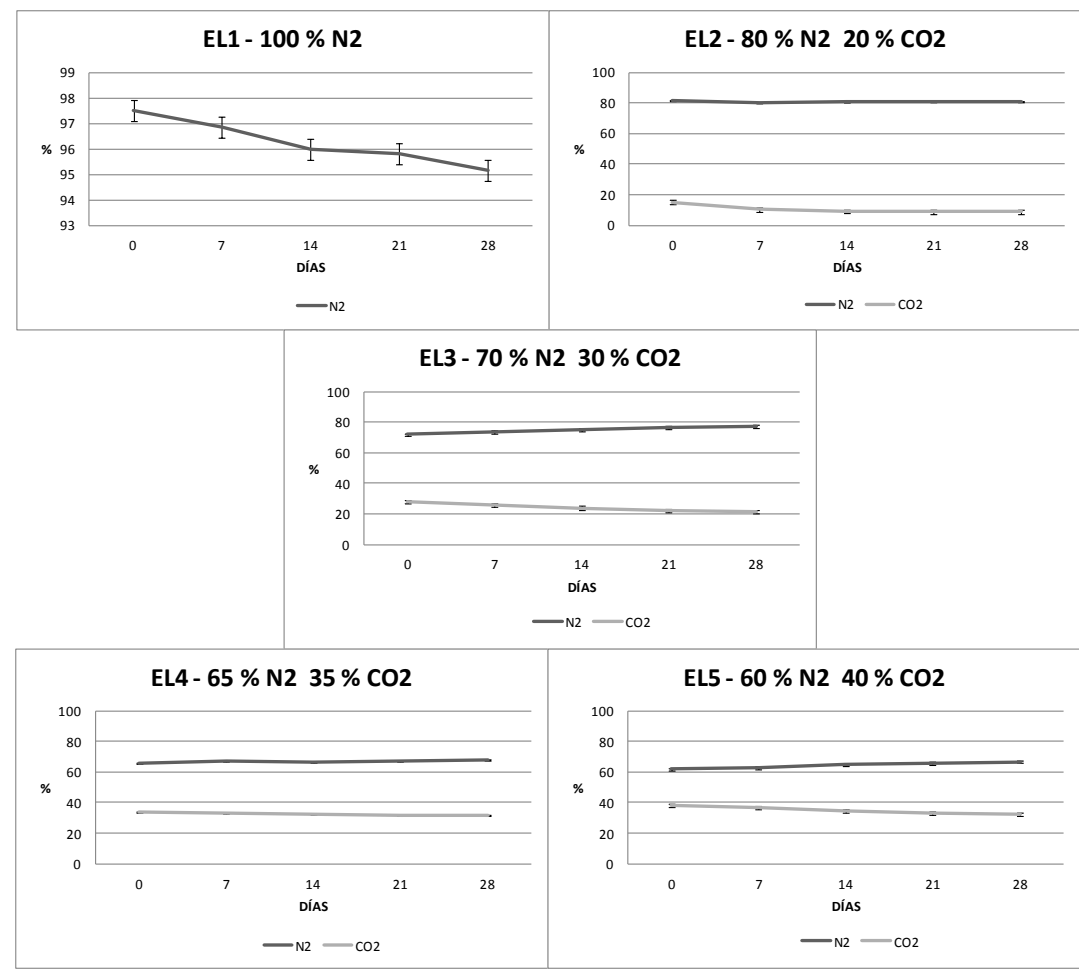

EL: envasado liofilizado.

Figura 1. Evolución de la concentración de gases de los tratamientos EL1, EL2, EL3, EL4 y EL5 durante el almacenamiento.

Tabla 2. Efecto del envasado en atmosfera modificada sobre el pH de las muestras de los tratamientos EL1, EL2, EL3, EL4 y EL5.

\begin{tabular}{|c|c|c|c|c|c|}
\hline \multirow[b]{2}{*}{ Días } & \multicolumn{5}{|c|}{ Condiciones de envasado } \\
\hline & EL1 $\left(100 \% \mathbf{N}_{2}\right)$ & $\begin{array}{c}\mathrm{EL2}\left(\mathbf{8 0} \% \mathrm{~N}_{2}\right. \\
\left.20 \% \mathrm{CO}_{2}\right)\end{array}$ & $\begin{array}{c}\operatorname{EL3}\left(70 \% \mathrm{~N}_{2}\right. \\
\left.30 \% \mathrm{CO}_{2}\right)\end{array}$ & $\begin{array}{c}\text { EL4 }\left(65 \% \mathrm{~N}_{2}\right. \\
\left.35 \% \mathrm{CO}_{2}\right)\end{array}$ & $\begin{array}{c}\operatorname{EL5}\left(60 \% \mathrm{~N}_{2}\right. \\
\left.40 \% \mathrm{CO}_{2}\right)\end{array}$ \\
\hline 0 & $5,55 \pm 0,10^{\mathrm{Aa}}$ & $5,60 \pm 0,04^{\mathrm{Ab}}$ & $5,72 \pm 0,01^{\mathrm{Ac}}$ & $5,86 \pm 0,19^{\mathrm{Ad}}$ & $5,69 \pm 0,05^{\mathrm{Ab}}$ \\
\hline 7 & $5,79 \pm 0,02 \mathrm{Ba}$ & $5,73 \pm 0,04^{\mathrm{Ba}}$ & $5,46 \pm 0,05^{\mathrm{Bc}}$ & $5,88 \pm 0,01 \mathrm{Ad}$ & $5,48 \pm 0,03^{\mathrm{Bc}}$ \\
\hline 14 & $5,76 \pm 0,04^{\mathrm{Ba}}$ & $5,82 \pm 0,03 \mathrm{Cb}$ & $5,63 \pm 0,02^{\mathrm{Cc}}$ & $5,75 \pm 0,19^{\mathrm{Ba}}$ & $5,66 \pm 0,04^{\mathrm{Ac}}$ \\
\hline 21 & $5,64 \pm 0,03 \mathrm{Ca}$ & $5,70 \pm 0,03 \mathrm{Bb}$ & $5,58 \pm 0,02 \mathrm{Dc}$ & $5,62 \pm 0,14 \mathrm{Ca}$ & $5,62 \pm 0,03$ Аa \\
\hline 28 & $5,66 \pm 0,03 \mathrm{Ca}$ & $5,75 \pm 0,03^{\mathrm{Bb}}$ & $5,74 \pm 0,07 \mathrm{Ab}$ & $5,59 \pm 0,02^{\mathrm{Cc}}$ & $5,75 \pm 0,04 \mathrm{Cb}$ \\
\hline
\end{tabular}

EL: envasado liofilizado. Letras mayúsculas en una misma columna indican para cada tratamiento diferencias significativas entre días. Letras minúsculas en una misma fila indican diferencias significativas entre tratamientos.

Aunque en las muestras de todos los tratamientos analizados a lo largo del envasado, el pH presentó diferencias significativas, estos resultados estuvieron en un rango de 5,5 - 5,8 similar a lo indicado por Swatland (1995) quien manifestó que el valor del pH para carnes normales está entre 5,4 a 5,7. Esto sugirió que las condiciones de envasado utilizadas no afectaron de forma negativa al $\mathrm{pH}$, coincidiendo con Barbosa-Cánovas y Vega-Mercado (2000) quienes concluyeron que un producto liofilizado correctamente envasado retiene durante más tiempo las propiedades químicas de la carne en estado fresco.
El color es una de las propiedades físicas que mayor deterioro presentó como consecuencia del proceso de la liofilización, es por ello que con el envasado en atmosfera modificada se buscó mantener y/o mejorar el color de las muestras tratadas.

El valor de L* (luminosidad) de las muestras liofilizadas de los tratamientos, fue parecido al inicio del envasado, de manera que las muestras presentaron luminosidad alta (Figura 2). A partir del día 7, las muestras de los

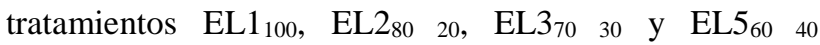
permanecieron sin cambios, mientras que las muestras del 
EL465 35 aumentaron ligeramente su valor de L*, presentando las muestras una tonalidad más clara. A lo largo del tiempo de envasado, el valor de $\mathrm{L}^{*}$ de las muestras liofilizadas de los tratamientos fue variando de tal forma que se vieron las diferencias entre tratamientos, es decir, las muestras pasaron de claras a ligeramente oscuras y viceversa según el tratamiento dentro de un rango de 50 a 60 de L*.

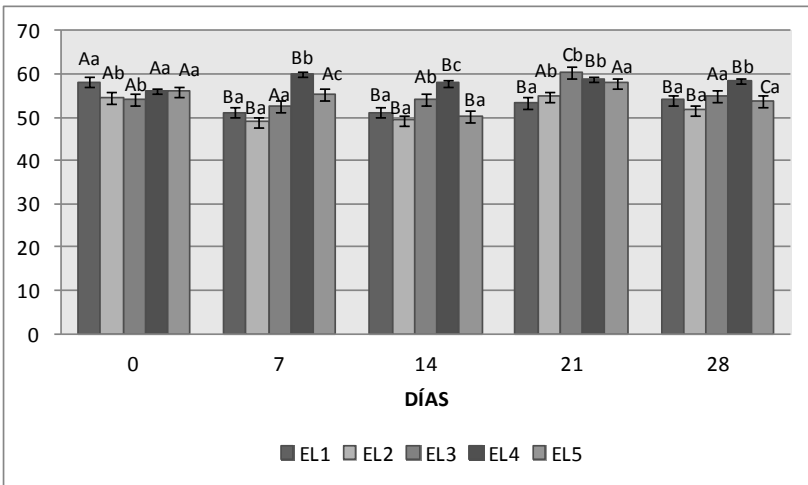

Figura 2. Efecto del $\mathrm{N}_{2}$ y $\mathrm{CO}_{2}$ sobre el valor de $\mathrm{L}^{*}$ de las muestras liofilizadas de los tratamientos EL1 $1_{100}$, EL2 80 20, EL3 70 30, EL465 35 y EL560 40. EL: envasado liofilizado. Letras mayúsculas indican, para cada tratamiento, diferencias significativas entre días. Letras minúsculas indican, para cada día, diferencias significativas entre tratamientos. Las barras de error representan el intervalo de confianza $(95 \%)$ para la media.

No obstante las muestras que mantuvieron mejor sus características iniciales de luminosidad hasta el final del tiempo de envasado fueron las muestras de los tratamientos EL1 $\left(100 \% \mathrm{~N}_{2}\right)$ y EL4 $\left(70 \% \mathrm{~N}_{2}-30 \% \mathrm{CO}_{2}\right)$. El efecto de la concentración de $\mathrm{CO}_{2}$ en el valor de $\mathrm{L}^{*}$ (luminosidad) de las muestras liofilizadas fue negativo; siendo aún más evidente el efecto negativo de éste gas en el valor de a* (rojo) de las muestras ya que se observó disminución de la tonalidad roja de las muestras liofilizadas (Figura 3).

Sin embargo, las muestras del tratamiento EL1 $1_{100}$ que sólo llevaron $\mathrm{N}_{2}$ fueron más rojas a lo largo del almacenamiento. Esto pudo deberse a la ausencia de $\mathrm{CO}_{2}$, ya que a este gas se le atribuyen reacciones adversas del color por altas concentraciones (Parry 1995; García 2006).

Las muestras del EL2 $\left(\begin{array}{llll}80 \% & \mathrm{~N}_{2}-20 \% & \mathrm{CO}_{2}\end{array}\right)$ también conservaron mejor la tonalidad roja (a*) durante el tiempo de envasado, ya que se observa más rojas al final del envasado que las muestras de los otros tratamientos, lo que pudo deberse a la baja concentración de $\mathrm{CO}_{2}$, tal como indicaron Parry (1995) y García (2006).

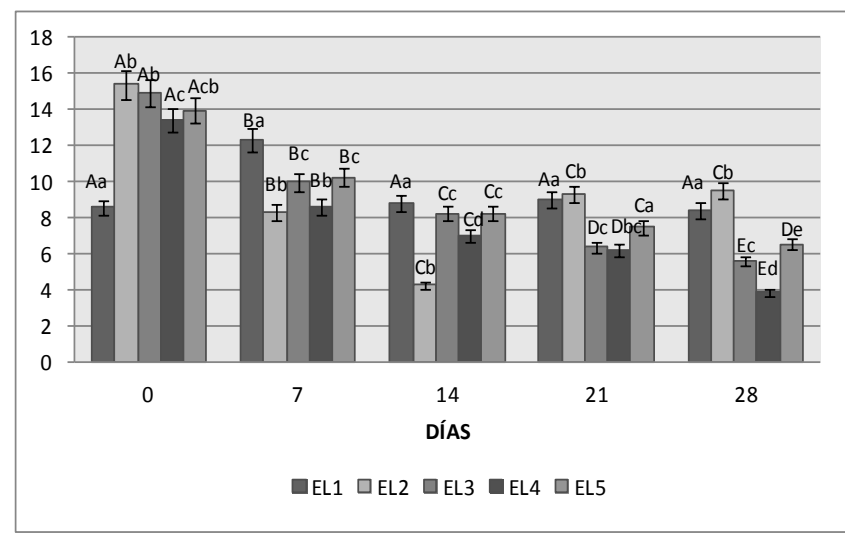

Figura 3. Efecto del $\mathrm{N}_{2}$ y $\mathrm{CO}_{2}$ sobre el valor de a* de las muestras liofilizadas de los tratamientos EL1 $_{100}$, EL2 80 20, EL3 70 30, EL465 35 y EL560 40. EL: envasado liofilizado. Letras mayúsculas indican, para cada tratamiento, diferencias significativas entre días. Letras minúsculas indican, para cada día, diferencias significativas entre tratamientos. Las barras de error representan el intervalo de confianza $(95 \%)$ para la media.

En cuanto al valor de $\mathrm{b}^{*}$ (amarillo) de las muestras liofilizadas del tratamiento EL1 se observa que hubo diferencias significativas respecto a los otros tratamientos el día 0 (Figura 4).

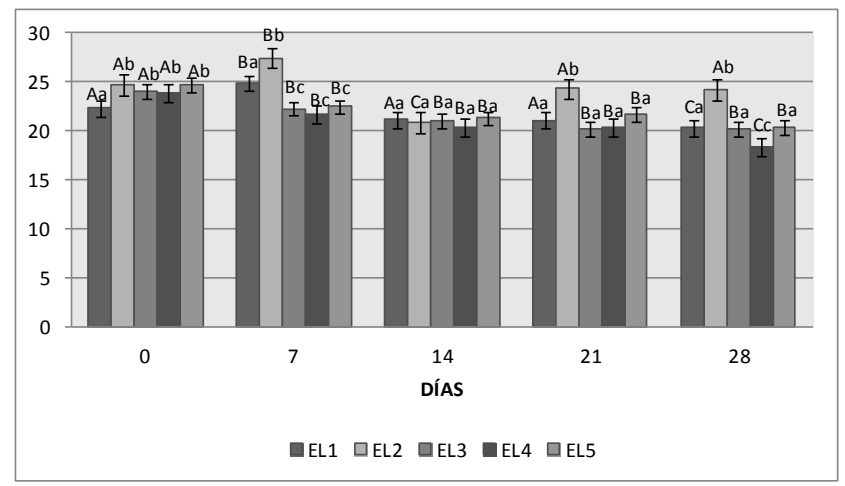

Figura 4. Efecto del $\mathrm{CO}_{2}$ sobre el valor de $b^{*}$ de las muestras liofilizadas de los tratamientos $\mathrm{EL}_{100}, \mathrm{EL} 2_{80} 20, \mathrm{EL} 3{ }_{70}$ 30, EL465 35 y EL560 40. EL: envasado liofilizado. Letras mayúsculas indican, para cada tratamiento, diferencias significativas entre días. Letras minúsculas indican, para cada día, diferencias significativas entre tratamientos. Las barras de error representan el intervalo de confianza $(95 \%)$ para la media.

Inicialmente todos los valores de $b^{*}$ fueron altos en todos los tratamientos, posteriormente, fue disminuyendo 
ligeramente, pero aun así las muestras fueron amarillas debido a que las muestras desde el principio fueron muy claras en cuanto a luminosidad y también por la disminución de la tonalidad roja a lo largo del tiempo de almacenamiento.

El valor de la tonalidad amarilla fue más alto en las muestras del tratamiento EL2 2020 a partir del día 7, disminuyendo el día 14 y volviendo a aumentar el resto de días.

En cuanto a la dureza obtenida a partir de la fuerza máxima de compresión, las muestras de los tratamientos EL1 100 y EL2 8020 inicialmente presentaron mayor fuerza de compresión que el resto, pero en los días posteriores la dureza disminuyó en las muestras de ambos tratamientos, aunque el último día de almacenamiento las muestras del tratamiento EL2 8020 fueron más duras que las del resto de tratamientos (Figura 5).

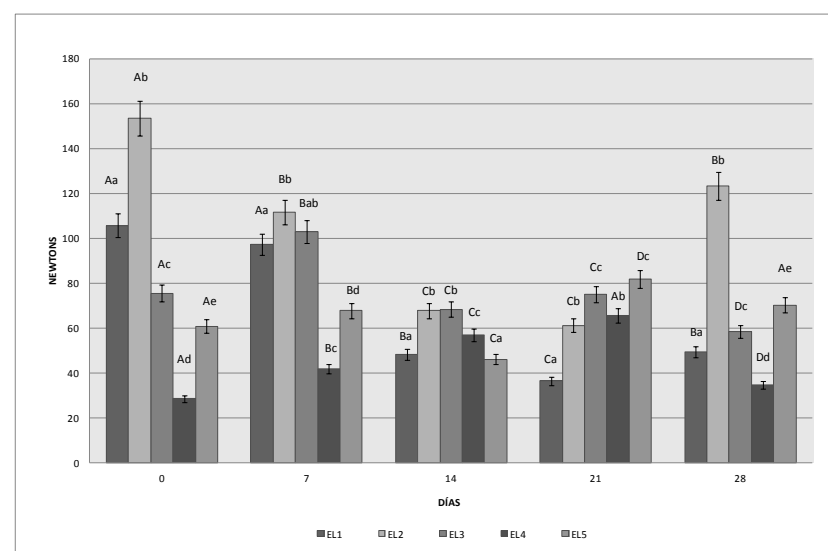

Figura 5. Evolución de la dureza de las muestras de los tratamientos EL1 100, EL2 80 20, EL3 70 30, EL465 35 y EL560 40. EL: envasado liofilizado. Letras mayúsculas indican, para cada tratamiento, diferencias significativas entre días. Letras minúsculas indican, para cada día, diferencias significativas entre tratamientos. Las barras de error representan el intervalo de confianza $(95 \%)$ para la media.

Las muestras del tratamiento EL465 35 inicialmente fueron las que menor dureza presentaron; sin embargo, durante los siguientes días de envasado (días 7, 14 y 21) la dureza de las muestras fue aumentando progresivamente, volviendo a disminuir la dureza de las muestras el día 28 de almacenamiento, lo que pudo deberse a que el día 28 la concentración de $\mathrm{N}_{2}$ fue en aumento mientras que el $\mathrm{CO}_{2}$ disminuyó.
Asimismo, la dureza de las muestras de los tratamientos EL3 $3_{70} 30$ y EL465 35, fue inicialmente baja y durante los siguientes días de almacenamiento fue en aumento, aunque siguió estando en un rango de fuerza de compresión baja.

Respecto a las propiedades sensoriales, el aspecto de las muestras EL2 8020 y el EL465 35 se mantuvo mejor y estable a lo largo del tiempo de almacenamiento. En el caso del EL2 8020 coincidió con la evolución de la tonalidad roja de las muestras (Figura 6); el aspecto es un atributo de impresión visual por lo tanto la coloración influye en la valoración de los jueces. Esto concuerda con lo indicado por Shafiur Rahman (2007), en que el envasado es una barrera que mantiene muy bien la calidad sensorial de los productos respecto al aspecto.

Según estos resultados, una atmosfera rica sólo en $\mathrm{N}_{2}$ conserva peor las características de impresión visual como el aspecto, ya que las muestras del tratamiento EL1 $1_{100}$ fueron consideradas con peor aspecto el día 28 de almacenamiento.

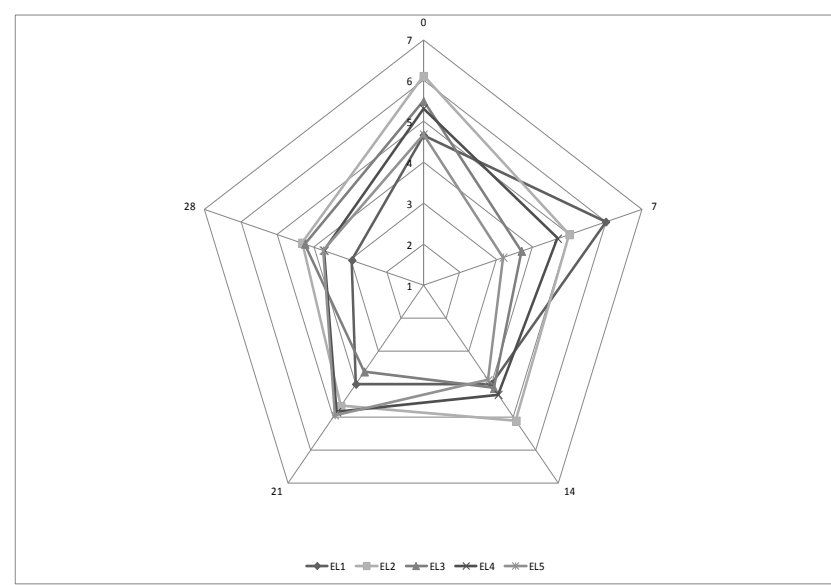

Figura 6. Evolución del aspecto de las muestras de los tratamientos EL1 100, EL280 20, EL3 70 30, EL465 35 y EL560 40. EL: envasado liofilizado.

Así mismo, en cuanto al atributo dureza de perfil de textura fueron las muestras del tratamiento EL465 35 las que mejor valoración obtuvieron, siendo calificadas como blandas. Estas características se mantuvieron ligeramente estables a lo largo del tiempo de almacenamiento. Del mismo modo, los resultados obtenidos instrumentalmente por las muestras de éste tratamiento concuerdan con la calificación dada por los jueces (Figura 7).

El día 28 de almacenamiento las muestras de los

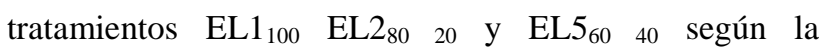
valoración de los jueces fueron las más duras, estas 
calificaciones sensoriales concuerdan con los resultados instrumentales obtenidos.

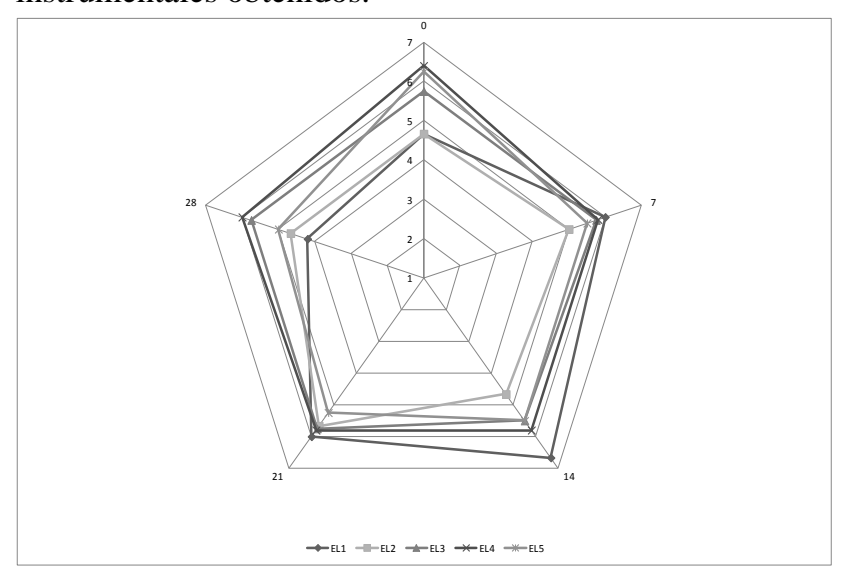

Figura 7. Evolución de la dureza de las muestras de los tratamientos EL1 100, EL280 20, EL370 30, EL465 35 y EL560 40. EL: envasado liofilizado.

\section{CONCLUSIONES}

A partir de los resultados obtenidos se puede afirmar que el tratamiento EL465 35 es el que mejor conservó las características físicas, químicas y sensoriales de las muestras de ternera liofilizadas durante el tiempo de almacenamiento.

El tratamiento EL2 8020 resulta mejor en la conservación de la tonalidad roja ( $\left.a^{*}\right)$ de las muestras y por lo tanto presentan un aspecto mejor valorado. Las muestras del tratamiento EL465 35 también presentan buen aspecto en cuanto al mismo atributo.

Con todos estos resultados se concluye que los tratamientos EL2 8020 y EL465 35 son las atmosferas más adecuadas para la conservación de carne de ternera liofilizada.

\section{AGRADECIMIENTOS}

A la Facultad de Ciencias Agrarias de la Universidad Nacional de Asunción, Paraguay y a la Universidad Pública de Navarra, España. A la Fundación Carolina y al Matadero de Pamplona, España.

\section{REFERENCIAS BIBLIOGRAFIAS}

Babić, J; Cantalejo, MJ; Arroqui, C. 2009. The effects of freeze-drying process parameters on Broiler chicken breast meat. LWT - Food Science and Technology 42:1325-1334.
Barbosa-Cánovas, GV; Vega-Mercado, H. 2000. Deshidratación de alimentos. Zaragoza, ES, Acribia. $314 \mathrm{p}$.

García Iglesias, E; Gago Cabezas, L; Fernández Nuevo, JL. 2006. Tecnologías de envasado en atmósfera protectora: informe de vigilancia tecnológica. Madrid, ES, Elecé. 143 p.

Hunt, MC; Anton, J; Benedict, RC; Conforht, CR; Jeremiath, DP. 1991 AMSA guidelines for meat color evaluation. In Proccedings 44 Annual Reciprocal Meat Conference (1991, Manhattan, US). US, Kansas State University. p. 3-17

Lyon, BG; Lyon, CE; 1991. Research note: shear value ranges by Instron Warner: bratzler and simple: blade allo: kramer devices that correspond to sensory tenderness. Poultry Science 70:188-191.

Moller, JKS; Jensen, JS; Olsen, MB; Skibsted, LH, Bertelsen, G. 2000. Effect of residual oxygen on colour stability during chill storage of sliced pasteurised ham packaged in modified atmosphere. Meat Science 54:399-405.

Moller, JKS; Jakobsen, M; Weber, CJ; Martinussen, T; Skibsted, LH; Bertelsen, G. 2003. Optimisation of colour stability of cured ham during packaging and retail display by a multifactorial design. Meat Science 63:169-175.

Nannerup, LD; Jakobsen, M; Berg, F; Jensen; JS; Moller, JKS; Bertelsen, G. 2004. Optimizing colour quality of modified atmosphere packed sliced meat products by control of critical packaging parameters. Meat Science 68:577-587.

Parry, RT. 1995. Envasado de los alimentos en atmosfera modificada. Madrid, ES, Antonio Madrid Vicente, 331 p.

Pedrero, D; Pangborn, RM. 1989. Evaluación sensorial de los alimentos: métodos analíticos. Mexico, MX, Alhambra Mexicana. 251 p.

Shafiur Rahman, M. 2007. Handbook of food preservation. 2 ed. US, Taylor y Francis. 1068 p.

Swatland, HJ. 1995. Evaluación de la carne en la cadena de producción. Zaragoza, ES, Acribia. 333 p. 